\title{
A cooperative covering problem under disruption considering backup coverage
}

\author{
Ladan Hazrati Ashtiani $^{1}$, Mehdi Seifbarghy ${ }^{1}$ and Mahdi Bashiri ${ }^{2}$ \\ ${ }^{1}$ Department of Industrial Engineering, Alzahra University, Tehran, Iran \\ ${ }^{2}$ Department of Industrial Engineering, Faculty of Engineering, Shahed University.
}

\begin{abstract}
In this paper, we study the location of emergency centres considering cooperative and backup coverage while natural disasters occur which can result in facility disruption. In this regard, a reliable version of cooperative covering problem is presented considering two types of candidate sites, i.e., reliable and unreliable. To achieve a fortified system against disaster, reliable candidate sites are selected from areas which are far away from the disaster harms. Furthermore, backup coverage is considered to compensate unsatisfied coverage of the demand zones due to facility disruption. The performance of the model is investigated solving numerical examples with different approaches utilising commercial software. The results confirm accurate performance of the model. They also show that both facility failure and backup coverage considerations lead to a more efficient network by incurring some additional cost.
\end{abstract}

Keywords: Backup coverage, Cooperative covering, Disruption, Natural disaster, Reliability. 